\title{
Bilateral hip reconstruction improve hip stability, pelvic balance and mobility function in children with cerebral palsy
}

\author{
Thamkunanon Verasak* \\ Queen Sirikit National Institute of Child Health, Thailand
}

\begin{abstract}
Background: Bilateral hip reconstructive surgery for hip displacement of children with cerebral palsy still be contro-versial. The objective of this study to report the outcome of bilateral hip surgery with varus derotation and shortening of the proximal femur and Dega pelvic osteotomy in radiography and mobility function of children with spastic diplegia and quadriplegia.
\end{abstract}

Methods: We retrospectively reviewed 49 patients with spastic diplegia or quadriplegia who underwent bilateral hip reconstruction at minimum follow up of 24 months. Surgical outcomes were assessed on potential in correction of hip displacement by following migration percentage and pelvic obliquity by following migration percentage difference and pelvic obliquity angle. Mobility function improvement was assessed on the basis of sifting, standing and walking capa-bility before and after surgery. The effect of preoperative migration percentage, age at surgery, GMFCS level and pelvic obliquity angle on surgical outcome were assessed by logistic regression analysis.

Result: $85.7 \%$ of the patients became normal hip coverage ( $\mathrm{MP}<33 \%)$ and $45 \%$ of the patients who have pelvic obliq-uity became balance pelvis at 2 years after surgery. Migration percentage, migration percentage difference and pelvic obliquity angle reduced significantly. $71 \%$ of the patients had improvement in sitting, $51 \%$ improved in standing and $12 \%$ improved in walking function. Preoperative migration percentage was only influential risk factor with respect to postoperative outcome.

Conclusion: Bilateral hip reconstruction can correct hip displacement problem either bilateral or unilateral displace-ment into normal hip coverage by $90 \%$ and decrease pelvic obliquity significantly as well as improve remarkably in sitting and standing function of the patients with spastic diplegia and quadriplegia.

Level of evidence: Therapeutic study, Level IV

\section{Introduction}

The risk of hip displacement in cerebral palsy varies from $0 \%$ to $64 \%$ according to CP subtype and GMFCS level. Pa-tients with spastic quadriplegia or in GMFCS level V are at the highest risk [1]. Progression of hip displacement in-creased markedly with decreasing functional level and increasing initial hip displacement $[2,3]$. Severe hip displace-ment can cause pain, difficulty to ambulate, pelvic obliquity, loss of sitting balance and scoliosis, especially in asymmetrical hip displacement $[4,5]$.

Early detection and intervention through hip surveillance program can obviously prevent hip dislocation and improve quality of life [6]. Early soft tissues release can prevent progression of hip displacement when there is no bony deformi-ty, but bone reconstructive surgery can effectively correct most of hip displacement, improve hip function and relieve pain in long term outcome $[7,8]$.

One-stage hip reconstruction which comprises of femoral varus derotation, shortening osteotomy (VDRSO) and pelvic osteotomy is considered to be the most appropriate choice for reduction. There was about $10 \%$ of operated hips loss of reduction, progressive recurrence of coxa valga, however hip stability still was maintained at maturity in $90 \%$ of hips [9].

One issue that is controversial is whether there is a necessity for bilateral hip reconstruction in spastic diplegia or qua-driplegia with unilateral hip displacement. Contralateral hip displacement and ipsilateral failure are the common com-plication after unilateral reconstruction, the rate is up to $74 \%$ [10-12]. Prophylactic surgery in the contralateral hip isn't recommended in patients with no hip dysplasia and patients should be closely monitored during the first two postoperative years [13]. Some authors stated that bilateral hip reconstruction in non-ambulatory quadriplegic patients should be considered to maintain symmetry and pelvic alignment, even if the contralateral hip is no any hip displacement $[14,15]$.

This study aims to report the outcome of bilateral hip reconstruction with varus derotation and shortening of the prox-imal femur with or without Dega pelvic osteotomy in correcting hip displacement, pelvic obliquity and improving mo-bility function of children with spastic diplegia and quadriplegia.

\section{Material and methods}

Ethical approval for this study was permitted under the audit

Correspondence to: Thamkunanon Verasak, MD, Queen Sirikit National Institute of Child Health, Bangkok, Thailand, E-mail: sivtk@hotmail.com

Key words: bilateral hip reconstruction, cerebral palsy, hip stability, pelvic balance', mobility function

Received: April 05, 2017; Accepted: April 26, 2017; Published: April 29, 2017 
provisions of our local institutional reviews board. This study was a prospective cohort of patients with spastic diplegia and quadriplegia who underwent bilateral hip recon-structive surgery since 2011 at the Queen Sirikit National Institute of Child Health, Bangkok, Thailand.

The inclusion criterion for bilateral hip reconstruction in our clinic are patients with spastic diplegic or quadriplegic cerebral palsy who have at least one side of hip displacement (Migration Percentage $(16,17)$ $\geqq 40 \%$ ), hip abduction $<60$ in extension, coxa valga, and excessive femoral anteversion. The patients presented a minimum follow up period of 24 months with clinical and radiological documentation at pre-operative point, 1 year and 2 years post-operative point.

We exclude adolescent patients who have severe deformation of femoral head, structural scoliosis and unhealthy enough for operation.

\section{Surgical procedure}

All parents were explained about the details of surgery. Patients were provided with physiotherapy program and general health assessment before operation.

All patients were treated with varus derotation and shortening osteotomy of proximal femur by single team of surgeons. The proximal femur was fixed with a 100 degree fixed angle blade osteotomy hip plate after alignment of neck shaft angle was set about 110-120 degree. Range of motion of the hip was examined by manipulation and the migration percentage was assessed by intra operative radiography. If the migration percentage was still more than $30 \%$ and the acetabular index was more than 25 degree, Dega type pelvic osteotomy [16-19] would be performed. If the range of hip abduction was still less than 60 degree, percutaneous release of hip adductor longus muscle and more shortening of femur would be considered. In patients with high level of hip dislocation, open hip reduction might be considered if the hip couldn't be reduced by the former steps of surgery. Other soft tissue procedure including medial hamstring release or tendo-achillis lengthening would be performed if the patients had contracture.

Postoperative pain control with epidural anaesthesia or continuous intravenous morphine had been applied for 2 days after operation. Sporadic dose of morphine was given in the first week. Hip abduction cast was applied in some cases with open hip reduction and severe spastic muscle tone. All patients were given by the same physiotherapy program both preoperative and postoperative period.

\section{Outcome measurement}

Data recorded from each patient are general information, radiographic parameters and mobility status before surgery, 1 year and 2 years after surgery. The parameters which had been recorded before and after surgery consist of sex, age at surgery, GMFCS level, radiographic parameters including migration percentage (MP), migration percentage difference (MPD) and pelvic obliquity angle (POA), and mobility functional status.

Radiographic assessment was performed with use of Migration Percentage (MP) of Reimers [16], Migration Percentage Difference (MPD) and Pelvic Obliquity Angle (POA). The MP describe the subluxation of the femoral head out of the acetabulum relative to the width of femoral head [17]. The MPD is the difference between the MP of one hip and the MP of the other hip in the same patient. We believe that high percent of the MPD is relative to high degree of pelvic obliquity. The POA is an angle between the line of the lowest edge of both ischium and horizontal line. It imply degree of pelvic obliquity as well (Figures 1-3).

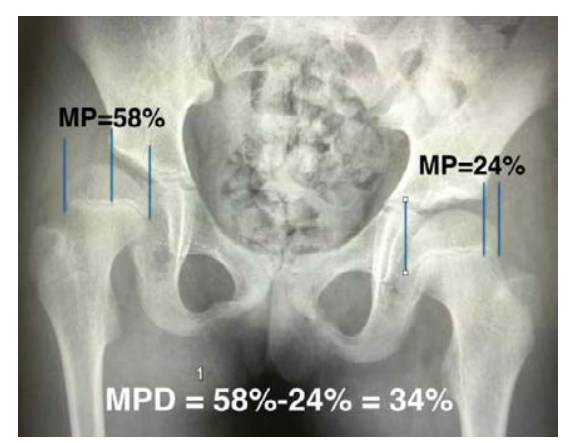

Figure 1. The migration percentage difference (MPD), the right hip $\mathrm{MP}=58 \%$ and the left hip MP $=24 \%$.the MPD $=58 \%-24 \%=34 \%$. High percent of MPD imply high degree of pelvic obliquity angle.

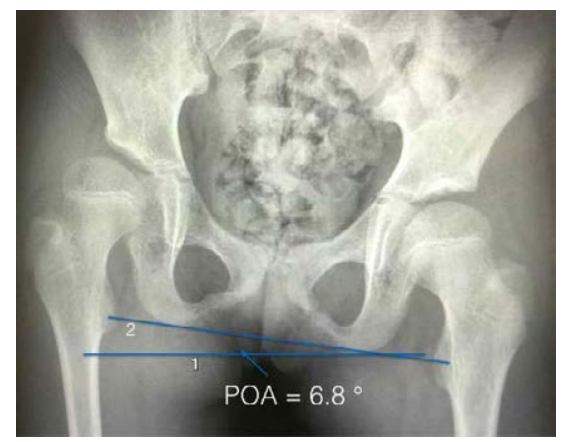

Figure 2. The pelvic obliquity angle (POA) consist of the 1st line(horizontal line) cross the second line(a line drawn from the lowest edge of both ischium).In this figure, POA is equal to 6.8 degree.The $\mathrm{x}$-ray film is belong to a 10 years-old girl with GMFCS level III. She walked with a walker frame in pattern of scissor gait and pelvic obliquity before operation. The right hip migrated $58 \%$ but the left hip migrated just $24 \%$ and the pelvic obliquity angle about 6.8 degree. The left hip had adduction, internal rotation contracture and the proximal femur showed coxa valga.

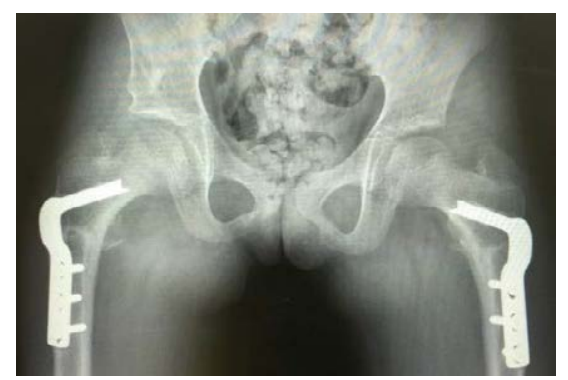

Figure 3. The same girl after 2 years of operation,the right hip was operated with proximal femur and pelvic osteotomy and the left hip was operated only with proximal femur osteotomy. The outcome showed that the right hip had $0 \%$ of MP and the left hip had $3 \%$ of MP.The MPD was equal to 3\% and the POA was about 2 degree. She could walk independently with no scissor gait pattern.

The mobility function form was designed to record and mobility functional status of cerebral palsy patients with the hip displacement at my clinic. The form consisted of 3 parts.

1. Sitting status, there are 3 levels of sitting status

i. Unable to sit : Patients are unable to maintain their trunk on a chair even though the chair has a back support.

ii. Sitting with back support: Patients can sit on a chair with back support.

iii. Sitting independently: Patients can sit independently on a chair.

2. Standing status, there are 4 levels of standing status. 
i. Unable to stand: Patients are unable to maintain their trunk on standing posture even though a person are holding on both arms.

ii. Standing with a person holding: Patients are able to stand with a person support by holding both arms.

iii. Standing with self holding: Patients are able to stand with holding a walker frame by themselves.

iv. Standing independently: Patients can stand independently.

3. Walking status, there are 3 levels of walking status.

i. Unable to walk: Patients are unable to walk even though they have a walker frame

ii. Walking with a walker frame: Patients can walk with a walker frame.

iii. Walking independently: Patients can walk independently.

\section{Evaluation}

To evaluate the effectiveness of correcting hip displacement, we defined the success of hip coverage as a migration percentage less than $33 \%[16]$.

In radiographic outcome, we monitored number of patients who had hip migration percentage more than $33 \%$ as well as high degree of pelvic obliquity before and 1 year, 2 years after surgery. We report number of patents whose hips become normal coverage and pelvis become symmetry.

Recorded data of migration percentage (MP), migration percentage difference (MPD) and pelvic obliquity angle (POA) were analyzed and reported as the mean and $95 \%$ confidence interval as well as comparison of the data between pre-operation,1st and 2nd year post-operation were analyzed by paired $t$-test. ( $p$ value of 0.05 was considered significant in all comparison). There are two groups of patients based on sides of hip displacement. Patients who had unilateral and bilateral hip displacement presented at pre-operation. The data between two groups were compared by unpaired t-test.

According to the success of hip coverage as MP less than 33\%, we compared the risk parameters including pre-operative MP, Age at surgery, GMFCS level and pelvic obliquity angle between the patients who had achievement in success and nonsuccess of hip coverage at 2 years after surgery to find out a potential risk factor by using logistic re-gression analysis statistic.

In mobility function outcome, we observed change of number of patients from pre-operation and 1st and 2nd year post-operation in sitting, standing and walking functional status.

\section{Result}

Forty nine patients who met the criteria underwent bilateral reconstruction by the same operative team and returned for following up at least 24 months. The average age of patients who had surgery was 7.63 years old (2.7-19.5). There were nineteen male and thirty female. Thirty five patients were classified in GMFCS level V, nine patients in GMFCS IV, four patients in level III and one in level II.

There were thirty eight patients with bilateral hips displacement and eleven patients with unilateral hip displacement, but all patients had bilateral hip contracture and coxa valga. The data is shown in table 1.

\section{Success in correcting unstable hip and pelvic obliquity}

All patients had abnormal pre-operative MP more than $33 \%$ but
Table 1. Patient demography.

\begin{tabular}{|l|l|}
\hline Follow up & minimum 24 months \\
\hline Number & 49 cases \\
\hline Number of operated hips & 98 \\
\hline Mean age(yr) & $7.63(2.7-19.5)$ \\
\hline Sex & $19(\mathrm{M}), 30(\mathrm{~F})$ \\
\hline GMFCS level (case) & $\begin{array}{l}\text { GMFCSII:1,GMFCS III:4 ,GMFCS IV: 9, } \\
\text { GMFCS V:35 }\end{array}$ \\
\hline Bilateral Hip subluxation(MP>33\%) & 38 cases \\
\hline Unilateral Hip subluxation & \\
\hline MP>33\% & 11 cases \\
\hline Pelvic Osteotomy & $40($ Dega) \\
\hline Proximal femur & $98($ VDRO+Shortening) \\
\hline
\end{tabular}

GMFCS: Gross Motor Functional Classification System

VDRO+ Shortening mean varus derotation osteotomy and shortening of proximal femur

after one year post operation the outcome showed 44 out of 49 patients had MP less than 33\%. Two years after surgery, 42 out of 49 patients had MP less than 33\%. 7 pa-tients still had MP more than $33 \%$ but less than $47 \%$. 2 patients had loss of successful MP correction from 1st year to 2nd years post operation.

Two radiological parameters were applied to assess degree of pelvic obliquity. The first parameter is the migration per-centage difference (MPD) and the second is the pelvic obliquity angle (POA). 17 patients had MPD less than $10 \%$ and 32 patients had MPD more than $10 \%$ before surgery. After one year post operation number of the patients with MPD more than $10 \%$ at pre-operation decreased from 32 to 12 patients or $64 \%$ reduction. After two years post-operation number of these patients decreased from 32 to 15 patients or $53 \%$.

Number of the patients with POA more than 0 degree before surgery decreased from 40 to 23 patients and 22 patients after one and two years post-operation consecutively. Data is shown on Table 2.

The functional mobility status of all patients recorded at preoperation, 1st year and 2nd years post-operation was re-ported into three parts. The first part is the sitting status. There were 12 patients in the unable to sit status before surgery but after 2 years of surgery only 4 patients still were in the unable to sit status. There were 27 patients in the sit with back support status before surgery but after 2 years only 10 patients still were in the sit with back support status and others became in the independent sit status. Number of the patients with independent sit status increased from 10 to 35 patients after 2 year of surgery. In sitting status part there were 35 out of 49 patients or $71 \%$ of patients had improve-ment of sitting function.

The second part is the standing status. 35 in 49 patients were in the unable to stand status before surgery but after 2 years of surgery the number decreased to 19 patients. In standing status part there were 25 out of 49 patients or $51 \%$ of patients improved in a standing status and no any patients worsen down. We noticed that all of the 25 patients who had improved in standing status part had good trunk control and were in the sit with back support or the independent sit status group before surgery.

The third part is the walking status. 44 in 49 patients were in the unable to walk status group before surgery but after 2 years of surgery the number decreased to 40 patients. There were only 6 patients out of 49 patents having improvement of walking function and we noticed that all 6 patients were in the independent sit status group. It implied that they had good trunk muscle control.

From this study, 43 in 49 or $87.7 \%$ of the patients had mobility 
Table 2. Alteration of number of the patients who had radiographic hip unstable, pelvic imbalance and functional mobility status after bilateral hip reconstruction.

\begin{tabular}{|c|c|c|c|}
\hline Condition & Number of patient at pre operation & $\begin{array}{l}\text { Number of patients at } \\
1 \text { year post operation }\end{array}$ & $\begin{array}{l}\text { Number of patients at } \\
2 \text { years post operation }\end{array}$ \\
\hline MP $>33 \%$ & 49 & 5 & 7 \\
\hline MP $>40 \%$ & 49 & 2 & 2 \\
\hline MPD $<10 \%$ & 17 & 37 & 34 \\
\hline MPD $>10 \%$ & 32 & 12 & 15 \\
\hline POA $=0$ & 9 & 26 & 27 \\
\hline POA $>0$ & 40 & 23 & 22 \\
\hline \multicolumn{4}{|l|}{ Ambulatory Status } \\
\hline Unable to sit & 12 & 6 & 4 \\
\hline Sit with back support & 27 & 9 & 10 \\
\hline Sit independent & 10 & 34 & 35 \\
\hline Unable to stand & 35 & 20 & 19 \\
\hline Stand with a person holding & 7 & 12 & 9 \\
\hline Stand with self holding & 5 & 12 & 16 \\
\hline Stand independent & 2 & 5 & 5 \\
\hline Unable to walk & 44 & 42 & 40 \\
\hline Walk with a walker frame & 4 & 4 & 6 \\
\hline Walk independent & 1 & 3 & 3 \\
\hline
\end{tabular}

MP: Migration Percentage, MPD: Migration Percentage Difference, POA: Pelvic Obliquity Angle

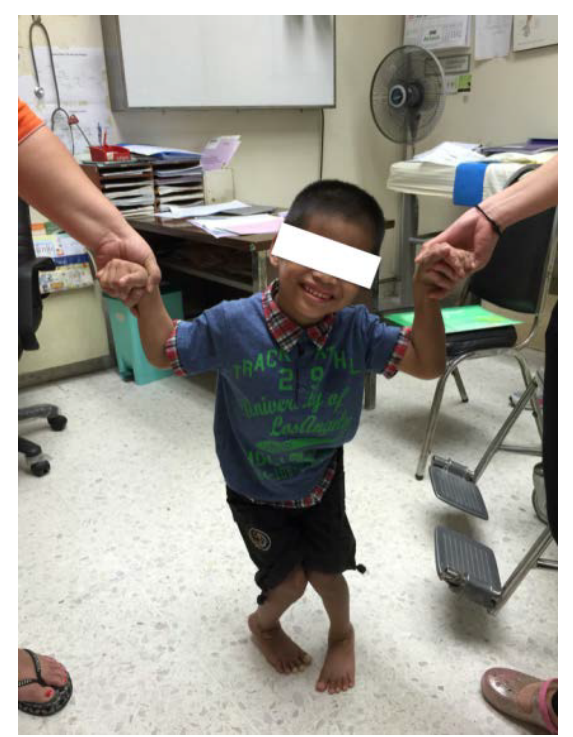

Figure 4. A 7 years old boy with spastic diplegia. Preoperative mobility status was independent sit, stand with a person hold and unable to walk.The hips had asymmetrical hip displacement.

function improvement after bilateral hip reconstruction. The improvement might be in sitting status part, standing status part or walking status part and no any patients were worsen. Data is shown in the Table 2 (Figures 4-7).

\section{Statistic analysis in 98 hips}

The mean value of preoperative migration percentage was $52.5 \%$ (16\%-100\%). The mean value of migration percen-tage significantly decreased to $12.3 \%(0 \%-44 \%)$ and $14.5 \%(0 \%-47 \%)$ after 1 and 2 years of surgery respectively. However the migration percentage increased a little bit $(2.2 \%)$ from the first to the second year as shown on Figure 8. Bilateral hip reconstructive surgery could correct hip displacement effectively although there was some loss of correc-tion in the second year.

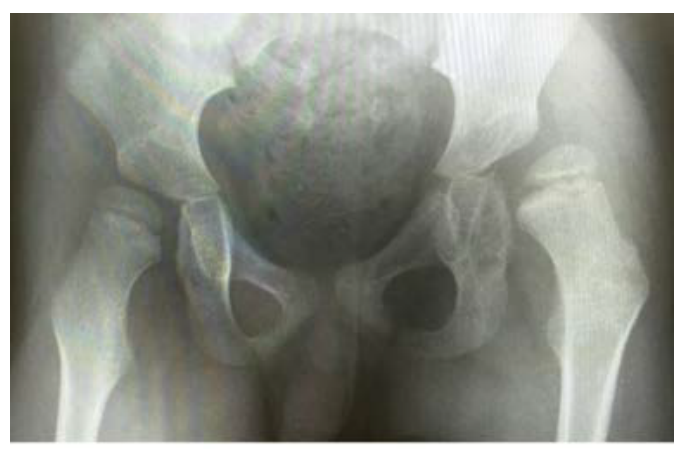

Figure 5. A 7 years old boy with spastic diplegia. Preoperative mobility status was independent sit, stand with a person hold and unable to walk.The hips had asymmetrical hip displacement.

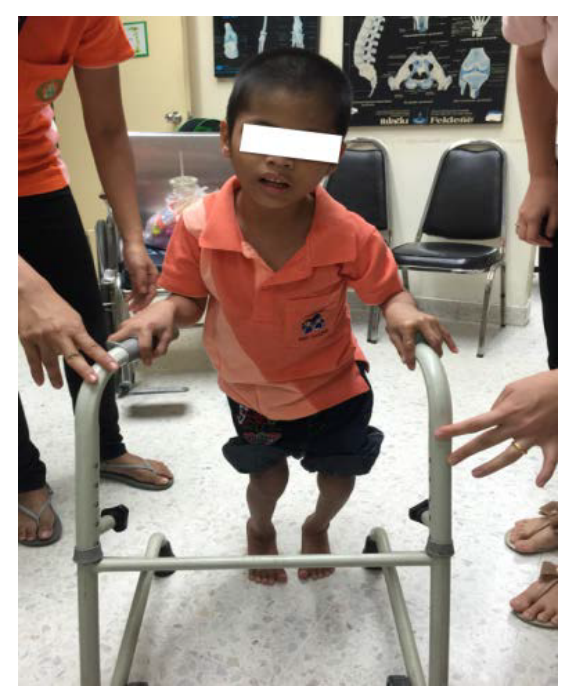

Figure 6. The same boy after 2 years of surgery,his mobility status was independent sit, stand with self holding and walk with a frame. The left hip underwent VDRO and the right hip underwent VDOR + Dega pelvic osteotomy.

The mean value of pre-operative migration percentage difference (MPD) was $23 \%(0 \%-72 \%)$ and it significantly de-creased to $8 \%(0 \%-$ 


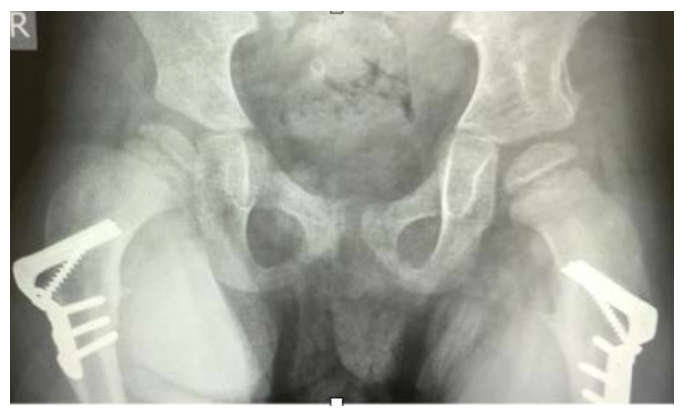

Figure 7. The same boy after 2 years of surgery,his mobility status was independent sit, stand with self holding and walk with a frame. The left hip underwent VDRO and the right hip underwent VDOR + Dega pelvic osteotomy.

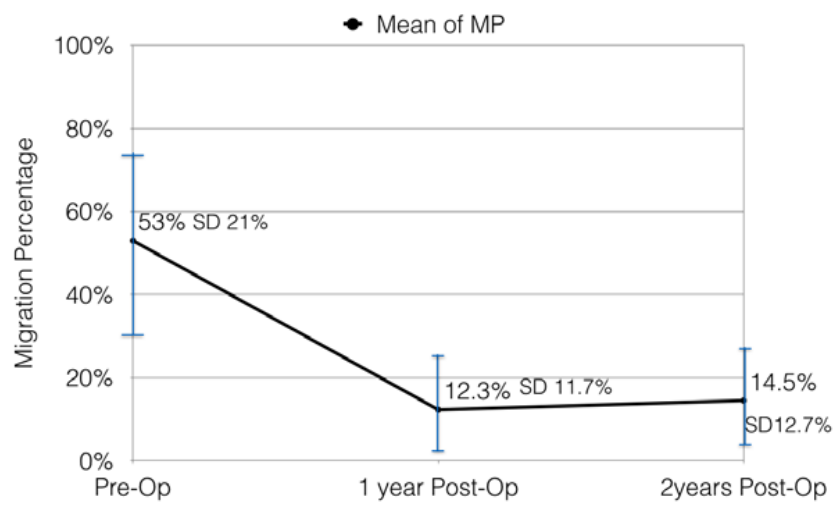

Figure 8. Graph show mean of migration percentage at pre-operation,1st year and 2nd year post-operation.

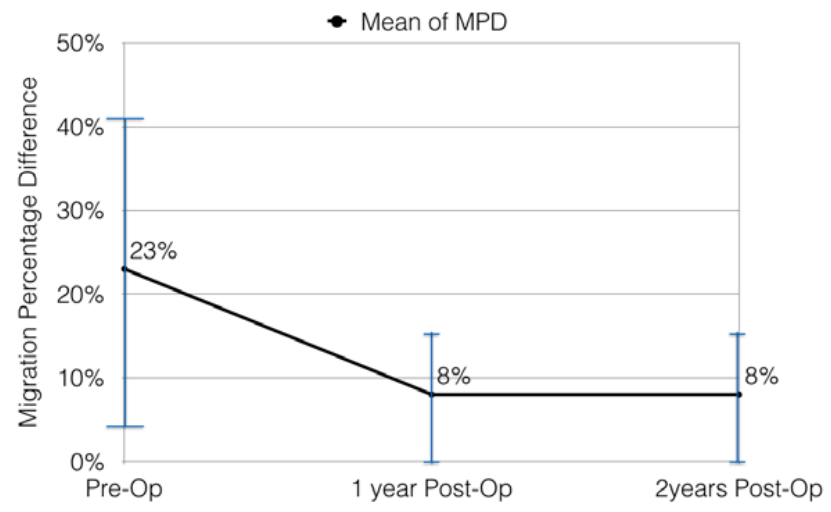

Figure 9. Graph show the migration percentage difference at pre-operation, 1st and 2nd year post-operation.

Table 3. Statistic Outcome after Bilateral Hip Reconstruction.

\begin{tabular}{|c|c|c|c|c|c|c|c|c|c|}
\hline & \multicolumn{3}{|c|}{$\begin{array}{c}\text { Pre Operation } \\
\text { Mean } \pm \text { SD }\end{array}$} & \multicolumn{3}{|c|}{$\begin{array}{c}\text { Post Operation:1 year } \\
\text { Mean } \pm \text { SD }\end{array}$} & \multicolumn{3}{|c|}{$\begin{array}{c}\text { Post Operation } 2 \text { years } \\
\text { Mean } \pm \text { SD }\end{array}$} \\
\hline & MP & MPD & $\mathrm{PT}$ & MP & MPD & $\mathrm{PT}$ & MP & MPD & PT \\
\hline Total 98 hips & $\mathbf{5 3} \% \pm 21 \%$ & $\mathbf{2 3} \% \pm 19 \%$ & $3.6^{\circ} \pm 4.5^{\circ}$ & $12.3 \% \pm 11.7 \%$ & $\mathbf{8 \%} \pm 8 \%$ & $1.6^{\circ} \pm 2.8^{\circ}$ & $\mathbf{1 4 . 5} \% \pm 12.7 \%$ & $8 \% \pm 9 \%$ & $1.5^{\circ} \pm 2.6^{\circ}$ \\
\hline $\begin{array}{l}\text { Bilateral subluxation group } \\
\text { ( } 38 \text { cases, } 76 \text { hips) }\end{array}$ & $\mathbf{5 4} \% \pm 19 \%$ & $17 \% \pm 15 \%$ & $2.3^{\circ} \pm 1.7 \circ$ & $13.7 \% \pm 12 \%$ & $\mathbf{8 \%} \pm 8 \%$ & $\mathbf{1} \circ \pm 1.4^{\circ}$ & $\mathbf{1 6 . 2} \% \pm 12.8 \%$ & $\mathbf{8 \%} \pm 9 \%$ & $\mathbf{1 . 1} 1^{\circ} \pm 1.6^{\circ}$ \\
\hline $\begin{array}{l}\text { Unilateral subluxation group } \\
\text { (11 cases,11 hips) }\end{array}$ & $68.5 \% \pm 17 \%$ & $44 \% \pm 20 \%$ & $8 \circ \pm 7 \circ$ & $7.9 \% \pm 11 \%$ & $6 \% \pm 9 \%$ & $3.6^{\circ} \pm 4.8^{\circ}$ & $\mathbf{8 \%} \pm 12 \%$ & $\mathbf{7 \%} \pm 9 \%$ & $2.9^{\circ} \pm 4.5^{\circ}$ \\
\hline $\begin{array}{l}\text { p value Bilateral vs Unilateral } \\
\text { group }\end{array}$ & $0.019^{*}$ & $0.001 * *$ & $0.04 *$ & 0.12 & 0.56 & 0.11 & 0.074 & 0.77 & 0.22 \\
\hline
\end{tabular}

41\%) after 1 and 2 years surgery as shown in Figure 9. In the same pattern the mean value of pre-operative pelvic obliquity angle (POA) was $3.6^{\circ}\left(0^{\circ}-30^{\circ}\right)$ and significantly decreased to $1.6^{\circ}\left(0^{\circ}-17^{\circ}\right)$ and $1.5^{\circ}\left(0^{\circ}-16^{\circ}\right)$ after 1 and 2 years of surgery consecutively. Outcome of both MPD and POA showed that bilateral hip surgery could correct pelvic obliquity. The data is shown in Table 3 (Figure 8-10).

\section{Comparison between bilateral and unilateral hip subluxation groups}

There were two subgroups of patients based on side of hip displacement. Bilateral hip displacement group that the pa-tients have migration percentage more than $33 \%$ both hips and unilateral hip displacement group that the patients have migration percentage more than $33 \%$ only one hip. We compared the outcome between 38 patients with bilateral hip displacement and 11 patients with unilateral hip displacement. The mean value of MP, MPD and POA of these two groups were significantly different before surgery which the mean values of the bilateral group were lower than the unilateral group as shown in Table 3. But after surgery the value of MP, MPD and POA between two groups weren't significantly different in statistic as shown in Table 3 and Figure 10. After bilateral hip reconstruction both unstable hip and pelvic obliquity problems could be corrected effectively in both bilateral and unilateral hip displacement patients.

\section{Logistic Regression analysis}

We had analysed the risk factors which might affect to success of correcting hip displacement. We set up a success of hip displacement correction as MP less than $33 \%$ at 2 nd post-operation. The factors that we tested were preoperative migration percentage between less than $80 \%$ and more than an equal $80 \%$, age at surgery between less than 5 years and more than and equal to 5 years old, GMFCS level between level II,III and IV,V and pelvic obliquity angle between 0 and more than 0 degree. Outcome showed only pre-operative MP significantly difference in statistic. Patients who had preoperative MP less than $80 \%$ were associated with more successful result as shown in Table 4. This data imply that we should do surgery before the hip established dislocation.

\section{Discussion}

Patients with cerebral palsy may present with symmetrical or asymmetrical hip displacement which may cause devel-opment of pelvic obliquity and scoliosis. Progressive deformity of hip, pelvis and trunk may develop continuously and contribute to difficulty in seating ,ambulation and handling [20-23]. One of common question which parent ask a treatment team is how much mobility function of the patients would be improved after surgery? Improvement of mobility function depend on three main factors: damage of mobility circuit in a brain, bone and joint deformity and muscle power. Developing of a surgical procedure that could effectively correct bone and joint

$*, * *$ symbol are statistically significant $\mathrm{p}<0.05$ 


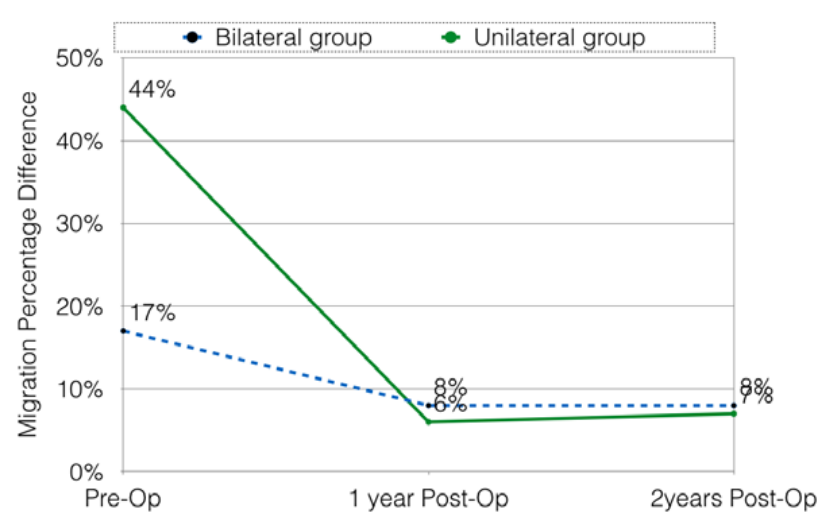

Figure 10. Graph show comparison of MPD between patients with bilateral and unilateral hip displacement at pre-operation, 1 st and 2nd year post-operation.

Table 4. Logistic regression analysis for risk factor of success hip coverage ( MP $<33 \%$ at 2nd year).

\begin{tabular}{|l|c|c|c|}
\hline Risk factor & P & $\mathbf{9 5 \%}$ CI & \\
\cline { 2 - 4 } & Lower & Upper \\
\hline $\begin{array}{l}\text { Pre Op MP } \\
<\mathbf{8 0 \%} \text { and }>\mathbf{8 0} \%\end{array}$ & $0.01 *$ & 1.54 & 25.60 \\
\hline $\begin{array}{l}\text { Age at surgery }<\mathbf{5} \\
\text { and }>\mathbf{5} \text { year }\end{array}$ & 0.792 & 0.224 & 3.03 \\
\hline $\begin{array}{l}\text { GMFCS II,III and } \\
\text { IV, }\end{array}$ & 0.282 & 0.88 & 2.03 \\
\hline POA 0 and > & 0.649 & 0.176 & 2.96 \\
\hline
\end{tabular}

*symbol are statistically significant $\mathrm{p}<0.05$

CI: Confidence Interval, MP: Migration Percentage, POA: Pelvic Obliquity Angle, GMFCS: Gross Motor Functional Classification System

deformity is an important duty of orthopaedic surgeon. One-stage hip reconstruction is the most appropriate and effective procedure that has been developed to reduce the hip displacement which include varus derotation and shortening osteotomy (VDRSO) of a proximal femur and Dega pelvic osteotomy [9].

Success of reconstructive hip surgery in correcting hip displacement depend on many factors such as level of GMFCS level, pre-operative hip displacement, surgical experience, and post operative care. Outcome of hip reconstruction have been reported in high success rate, up to $96 \%$ for the past five years $[9,24,25]$. However recurrent subluxation of oper-ated hip and progressive displacement of contralateral hip have been reported in high rate, up to $74 \%$ especially in un-ilateral hip reconstruction [10-12]. Nevertheless some authors [14,15] had recommend bilateral hip reconstruction in severe non ambulate CP patients who had hip displacement to balance pelvic asymmetry but it was a controversy issue due to difficult and surgical risk of bone reconstructive surgery. Some authors [26] recommend closely observe further migration of contralateral hip and early soft tissue release in clinical contracture patient. Therefore we believe that bilateral hip reconstruction could correct asymmetrical hip displacement, some of pelvic obliquity and prevent the risk of subsequent hip displacement as well as improve mobility function especially in patients with potential for ambulation.

In this study we had performed one stage bilateral hip reconstruction in spastic diplegia and quadriplegia who have bilateral brain involvement in order to show the outcome in correcting hip unstable, pelvic obliquity and mobility function improvement. The outcome of hip displacement correction to normal migration percentage show high success rate $(89.7 \%)$ in the first year and $85.7 \%$ in the second year post operation. There were only $4.5 \%$ of patients have recurrent subluxation which the migration percentage was mild grade (less than $47 \%$ ) and only 1 patients was re-operated because of progression of subluxation and clinical hip contracture in the third year. The mean of migration percentage decreased significantly from $53 \%$ in pre-operation to $12.3 \%$ in the first year and $14.5 \%$ in the second year post-operation. The first year result reflect to effectiveness of surgical procedure but after that result might be correlate to severity of spasticity, growth potential and post-operative care. We had tested risk factors (pre operative MP, age at surgery, GMFCS level and pelvic obliquity) by logistic regression analysis and found that only pre operative MP was significantly relative to the outcome. We recommend doing surgery before establishment of hip dislocation.

Pelvic obliquity derived from hip displacement might contribute to wind swept deformity, secondary scoliosis and diffi-cult in care. Balancing of both hips by correcting hip bone deformity and soft tissue contracture may help reduce pelvic obliquity. In this study we measure improvement of pelvic obliquity with migration percentage difference (MPD) and pelvic obliquity angle (POA). The mean of migration percentage difference decreased significantly from $23 \%$ in pre-operation to $8 \%$ in the first and second year after operation. The number of patients who had high value of MPD and POA reduced about $50 \%$ at the second year after surgery.

In comparison between unilateral and bilateral hip displacement, there are 38 patients bilateral hip displacement and 11 patients unilateral hip displacement before operation. The mean of migration percentage, migration percentage difference and pelvic obliquity angle of the unilateral hip displacement group were significantly higher than the bilateral hip displacement group at pre-operation but these differences were corrected into the same level, non-significance difference in statistic after surgery. Pelvic obliquity could be effectively corrected by bilateral hip reconstruction unless pelvic obliquity causes from supra pelvic origin.

How much of mobility function improvement after hip reconstruction in cerebral palsy is one of controversy issue and hard to predict the result because the main predictor of mobility function is severity of brain involvement. However good lever arm of the hips which surgeon could correct might help better control of lower extremity and trunk motion. Many authors have reported the better of patient care, rang of hip motion and relief of pain in long term outcome after reconstruction but no any detail of mobility improvement $[8,14,15]$. In this study we try to assess simply a mobility function of patients at pre and post operation. We found that most of the patients (71\%) had improvement in sitting function. Some patient became sit independently from pre-operation period they couldn't sit up. Asymmetrical hip displacement might contribute to pelvic obliquity and disturb siting function hence if we could correct asymmetrical hip displacement, siting function might be better. Although some patient with poor back muscle control couldn't sit inde-pendently, mobility of hip joint was given by surgery would help patients in positioning of siting with a support.

There were $51 \%$ of the patients have improvement in standing status by 1 or 2 level. Standing is required good lever arm of whole lower extremity particularly both hip joints and knees joint control. Some patient had a good trunk and hip control but their quadricep muscle and knee motion not good enough for standing so they couldn't stand up after hip surgery. We noticed that all of patients who were 
improved in standing had preoperative sitting status as sitting with back support or sitting independently. Because they had adequate trunk muscle control, so after hip surgery they would have a good potential for standing improvement.

function is the most complex motion required good lever arm of lower extremity, adequate muscle power and walking circuit in a brain but in cerebral palsy patients who have hip displacement usually are classified in GMFCS level IV or V that their brains have severe damage involving walking control circuit. It is hard to have walking control circuit remain function in these patients. In our study group, almost all $(89.7 \%)$ of patients were unable to walk before operation. After 2 years post operation there were only $12.2 \%$ or 6 patients have walking ability improvement and all of them had a good trunk muscle control or were in independent sitting status before operation. There were 2 patients who started with walking with a walker frame and after operation they could walk independently. Trunk muscle control can be assessed simply by showing capability of patient in sitting function. If the patients could sit independently or leaning with a back support, they have a good candidate for correcting lever arm of lower extremity especially hip displacement and we could hope them better in mobility function.

One of limitation of this study isn't a long term outcome but it could answer the effectiveness of bilateral hip recon-struction in radiological and functional outcome. Migration percentage difference, pelvic obliquity angle and mobility functional assessment is the parameters which have never reported in the literature, intra and inter observer reproduci-bility were not evaluated. Further assessment may be necessary.

\section{Conclusion}

Bilateral hip reconstruction can correct hip displacement problem either bilateral or unilateral displacement into normal hip coverage by $90 \%$ and decrease pelvic obliquity significantly as well as improve remarkably in sitting and standing function of the patients with spastic diplegia and quadriplegia.

\section{References}

1. Hägglund G, Lauge-Pedersen H, Wagner P (2007) Characteristics of children with hip displacement in cerebral palsy. BMC Musculoskelet Disord 8: 101. [Crossref]

2. Terjesen T (2012) The natural history of hip development in cerebral palsy. Dev Med Child Neurol 54: 951-957. [Crossref]

3. Pruszczynski B, Sees J, Miller F (2016) Risk Factors for Hip Displacement in Children With Cerebral Palsy: Systematic Review. J Pediatr Orthop 36: 829-833. [Crossref]

4. Ramstad K, Terjesen T (2016) Hip pain is more frequent in severe hip displacement: a population-based study of 77 children with cerebral palsy. J Pediatr Orthop B 25: 217-221. [Crossref]

5. Senaran H, Shah SA, Glutting JJ, Dabney KW, Miller F (2006) The associated effects of untreated unilateral hip dislocation in cerebral palsy scoliosis. J Pediatr Orthop 26: 769-772. [Crossref]

6. Hägglund G, Alriksson-Schmidt A, Lauge-Pedersen H, Rodby-Bousquet E, Wagner $\mathrm{P}$, et al. (2014) Prevention of dislocation of the hip in children with cere-bral palsy: 20 -year results of a population-based prevention programme. Bone Joint J 96-B: 15461552. [Crossref]

7. Spiegel DA, Flynn JM (2006) Evaluation and treatment of hip dysplasia in cerebra palsy. Orthop Clin North Am 37: 185-196. [Crossref]

8. Rutz E, Vavken P, Camathias C, Haase C, Jünemann S, et al. (2015) Long-term results and outcome predictors in one-stage hip reconstruction in children with cerebral palsy. J Bone Joint Surg Am 97: 500-506. [Crossref]

9. Mallet C, Ilharreborde B, Presedo A, Khairouni A, Mazda K, et al. (2014) One-stage hip reconstruction in children with cerebral palsy: long-term results at skeletal maturity. $J$ Child Orthop 8: 221-228. [Crossref]
10. Noonan KJ, Walker TL, Kayes KJ, Feinberg J (2000) Effect of surgery on the nontreated hip in severe cerebral palsy. J Pediatr Orthop 20: 771-775. [Crossref]

11. Canavese F, Emara K, Sembrano JN, Bialik V, Aiona MD, et al. (2010) Varus derotation osteotomy for the treatment of hip subluxation and dislocation in GMFCS level III to $\mathrm{V}$ patients with unilateral hip involvement. Follow-up at skeletal maturity. J Pediatr Orthop 30: 357-364. [Crossref]

12. Shukla PY, Mann S, Braun SV, Gholve PA (2013) Unilateral hip reconstruction in children with cerebral palsy: predictors for failure. J Pediatr Orthop 33: 175-181. [Crossref]

13. Abdo JCM, Forlin E (2016) Hip dislocation in cerebral palsy: evolution of the contralateral side after reconstructive surgery. Rev Bras Ortop 51: 329-332.

14. Owers KL, Pyman J, Gargan MF, Witherow PJ, Portinaro NM (2001) Bilateral hip surgery in severe cerebral palsy a preliminary review. J Bone Joint Surg Br 83: 11611167. [Crossref]

15. Barakat MJ, While T, Pyman J, Gargan M, Monsell F (2007) Bilateral hip reconstruction in severe whole-body cerebral palsy: ten-year follow-up results. $J$ Bone Joint Surg $\operatorname{Br}$ 89: 1363-1368. [Crossref]

16. Reimers J (1980) The stability of the hip in children. A radiological study of the results of muscle surgery in cerebral palsy. Acta Orthop Scand Suppl 184: 1-100. [Crossref]

17. Hägglund G, Lauge-Pedersen H, Persson M (2007) Radiographic threshold values for hip screening in cerebral palsy. J Child Orthop 1: 43-47. [Crossref]

18. Sales de Gauzy J (2010) Pelvic reorientation osteotomies and acetabuloplasties in children. Surgical technique. Orthop Traumatol Surg Res 96: 793-799. [Crossref]

19. Robb JE, Brunner RA (2006) Dega-type osteotomy after closure of the triradiate cartilage in non-walking patients with severe cerebral palsy. J Bone Joint Surg Br 88: 933-937. [Crossref]

20. Patel J, Shapiro F (2015) Simultaneous progression patterns of scoliosis, pelvic obliquity, and hip subluxa-tion/dislocation in non-ambulatory neuromuscular patients: an approach to deformity documentation. J Child Orthop 9: 345-356. [Crossref]

21. Hodgkinson I, Bérard C, Chotel F, Bérard J (2002) [Pelvic obliquity and scoliosis in non-ambulatory patients with cere-bral palsy: a descriptive study of 234 patients over 15 years of age]. Rev Chir Orthop Reparatrice Appar Mot 88: 337-341. [Crossref]

22. Morrell DS, Pearson JM, Sauser DD (2002) Progressive bone and joint abnormalities of the spine and lower extremities in cerebral palsy. Radiographics 22: 257-268. [Crossref]

23. Abel MF, Blanco JS, Pavlovich L, Damiano DL (1999) Asymmetric hip deformity and subluxation in cerebral palsy: an analysis of surgical treatment. J Pediatr Orthop 19: 479-485. [Crossref]

24. Reidy K, Heidt C, Dierauer S, Huber H (2016) A balanced approach for stable hips in children with cerebral palsy: a combination of moderate VDRO and pelvic osteotomy. $J$ Child Orthop 10: 281-288. [Crossref]

25. Zhang S, Wilson NC, Mackey AH, Stott NS (2014) Radiological outcome of reconstructive hip surgery in children with gross motor function classification system IV and V cerebral palsy. J Pediatr Orthop B 23: 430-434. [Crossref]

26. Dhawale AA, Karatas AF, Holmes L, Rogers KJ, Dabney KW, et al. (2013) Long-term outcome of reconstruction of the hip in young children with cerebral palsy. Bone Joint J 95-95B: 259-65. [Crossref]

Copyright: (C2017 Verasak T. This is an open-access article distributed under the terms of the Creative Commons Attribution License, which permits unrestricted use, distribution, and reproduction in any medium, provided the original author and source are credited. 